\title{
Within-subject repeatability of scotopic ERG
} measurements

\author{
WDH Gillan \\ Department of Optometry, University of Johannesburg, PO Box 524, Auckland Park, 2006 South Africa
}

Received 28 November 2011; revised version accepted 28 May 2012

<wgillan@uj.ac.za>

\begin{abstract}
The repeatability of data collected during clinical or research measurements needs to be considered by clinicians and researchers. Conclusions made relating to such measurements might be influenced improperly if an instrument proves to not be repeatable or accurate. The aim of this study was to investigate the within-subject repeatability of scotopic electroretinogram (ERG) measurements. Ten ERG
\end{abstract}

recordings were recorded for each eye from six volunteers using a CSO Retimax electrophysiology system. Only one eye was dilated. The results of this study support other researchers in their cautious approach regarding repeated ERG measurements. (S Afr Optom 2012 71(2) 64-69)

Key Words: Electroretinography, electroretinograms, electrophysiology, repeatability, retinal function.

\section{Introduction}

The fact that a flash of light presented to an eye will elicit a characteristic electrical response from such an eye has been accepted for over a century ${ }^{1,2}$. The characteristic electrical response is known as the electroretinogram (ERG). The ERG is variously defined as: "Recording of mass electrical response of the retina when it is stimulated by light" 3 or "The action potential recorded from the surface of the eyeball and originated by a pulse of light"4. The ERG can be used in numerous investigations of retinal function like: the effects of amblyopia on the retina, myopia effects on the retina, disease, genetic influences and ganglion cell function among others ${ }^{2}, 5,6$. An ERG can take various forms (for example: rod ERG, standard combined ERG, oscillatory potential, single-flash cone ERG, multifocal ERG, scotopic threshold response ERG and others) ${ }^{4}$. As with any such test the repeatability of the data acquired is an important aspect influencing the confidence with which clinicians and researchers use such data. The effects of clinical disease, therapeutic intervention, research intervention or time effects on ERG data need to be able to be distinguished from any possible inherent variability within the instrument (technological error). Normally present, physiological variation (biological error) also needs to be distinguished from "treatmentrelated" change. Hopkins 7 states that "measurement error" encompasses all types of variation in measurements from whatever source. Repeatability is defined as: "closeness of the agreement between the results of successive measurements of the same measurand carried out under the same conditions of measurement" 8 . The conditions of repeatability include: "the same measurement procedure, the same observer, the same instrument used under the same conditions, the same location, and, the repetition needs to be over a short period of time" 8 . Bland and Altman", 10 emphasize that multiple measurements (of the same measure) taken of the same subject will not be the same and also that the errors that occur in measurements need to be quantified. Having some understanding of the error within measurements enables one to decide if changes in measurements are real. As Hess ${ }^{11}$ et al state "normal eyes display a substantial degree of variability across subjects for psychophysical thresholds as well as pattern ERG amplitude". 
The repeatability of various aspects of the ERG has been investigated by numerous authors. The influence of different types of electrode on repeatability have been reported ${ }^{12,13}$. The effects of digital filtering on the intra-session repeatability of the pattern ERG have been investigated, at least partly due to the opinion that inadequate attention has been given to pattern ERG repeatability ${ }^{14}$. Holopigian et al ${ }^{15}$, reporting on the variability of the pattern ERG, state that one must be careful in evaluating data from this technique and that caution needs to be taken in its clinical use. Holopigian et al ${ }^{15}$ were of this opinion after they found that intra-session variability in pattern ERG measurements "remained high". The terms variation and repeatability seem to be used interchangeably by various authors. In this study the premise that excess variation in measurements is an indication of poor repeatability of those measurements is accepted.

The aim of this study was to investigate the within-subject repeatability of scotopic (rod) flash ERG measurements.

\section{Method}

Six volunteers gave their informed consent to participate in this study. All subjects were treated according to the tenets of the declaration of Helsinki. Subjects were final year optometry students of the University of Johannesburg aged between 21 and 23 years. Three females and three males participated. Measurements were obtained using a CSO Retimax Plus electrophysiology system. Scotopic flash ERG recordings were obtained from each subject. As flash ERG measurements were obtained subjects wore no refractive compensation ${ }^{1}$. Each flash was presented at $3 \mathrm{~cd} / \mathrm{m}^{2}$, at $0.5 \mathrm{~Hz}$ and with an acquisition time of 250 ms. Five events (flashes) were used to generate each ERG. Mydriasis was induced in one eye using mydriacyl (each subject chose the eye they wanted dilated). Measurements were obtained from each subject at approximately the same time of day. Subjects were dilated as this study was multi-faceted. One aspect of the study was to compare the effects of dilation versus non-dilation on the ERG while the other was to investigate the repeatability of multiple flash ERG recordings. Before being seated in the dark each subject had the relevant electrodes attached to the lateral side of each orbit with a reference electrode attached above the nose on the forehead. Prior to attaching the electrodes the appropriate patch of skin was cleaned with Nuprep abrasive gel. Each electrode was attached using Ten ${ }^{20}$ conductive EEG paste and micropore surgical tape. The H-R ocular electrode was attached later after dark adaptation had taken place. Each subject sat in a dark room for 25 minutes to allow dark adaptation to take place. At the appropriate time, using red light in the darkened room, the H-R electrodes were applied to each eye. The impedance of each electrode was then checked and had to be below 10 Ohms (according to the manufacturer's instructions). At this point the ERG was started and was conducted in a dark room. A total of ten scotopic flash ERG recordings were produced for each subject (the ERGs for both eyes were recorded simultaneously resulting in a total of twenty ERG plots per subject).

\section{Results}

Figure 1 gives an example of an ERG recording that was obtained during this study and ten such recordings were produced for each subject. Included are the ERG plots and the associated amplitudes and implicit times for the B and C peak/troughs of each plot. The amplitudes and implicit times were used as data in the repeatability investigation in this study. The $\mathrm{B}_{1}$ peak and $\mathrm{C}_{1}$ trough are indicated on each ERG plot. Table 1 shows the mean, minimum measurement, maximum measurement and standard deviation of the B1 amplitudes (in micro-volts) of the ten recordings obtained for each eye of each subject.

Table 1: The mean $\mathrm{B}_{1}$ amplitude, minimum measurement, maximum measurement and standard deviation of ten recordings for each eye of each subject are given. Values are recorded in micro-volts. UD indicates the un-dilated eye while D indicates the dilated eye for each subject (this applies to all tables unless stated otherwise).

\begin{tabular}{l|l|l|l|l} 
Subject & Mean & Minimum & Maximum & Standard deviation \\
\hline 1UD & 159.67 & 97.61 & 189.91 & 28.94 \\
1D & 207.07 & 184.19 & 228.67 & 16.92 \\
2UD & 109.81 & 104.30 & 119.03 & 4.42 \\
2D & 149.61 & 144.77 & 154.46 & 3.06 \\
3UD & 42.47 & -0.51 & 61.88 & 16.57 \\
3D & 56.15 & 38.24 & 77.75 & 11.03 \\
4UD & 149.06 & 58.83 & 225.98 & 43.23 \\
4D & 191.23 & 48.29 & 249.95 & 76.01 \\
5UD & 194.91 & 178.92 & 212.07 & 10.10 \\
5D & 140.92 & 129.88 & 160.29 & 8.15 \\
6UD & 103.53 & 31.25 & 156.02 & 42.01 \\
6D & 152.48 & 114.33 & 177.41 & 19.35
\end{tabular}




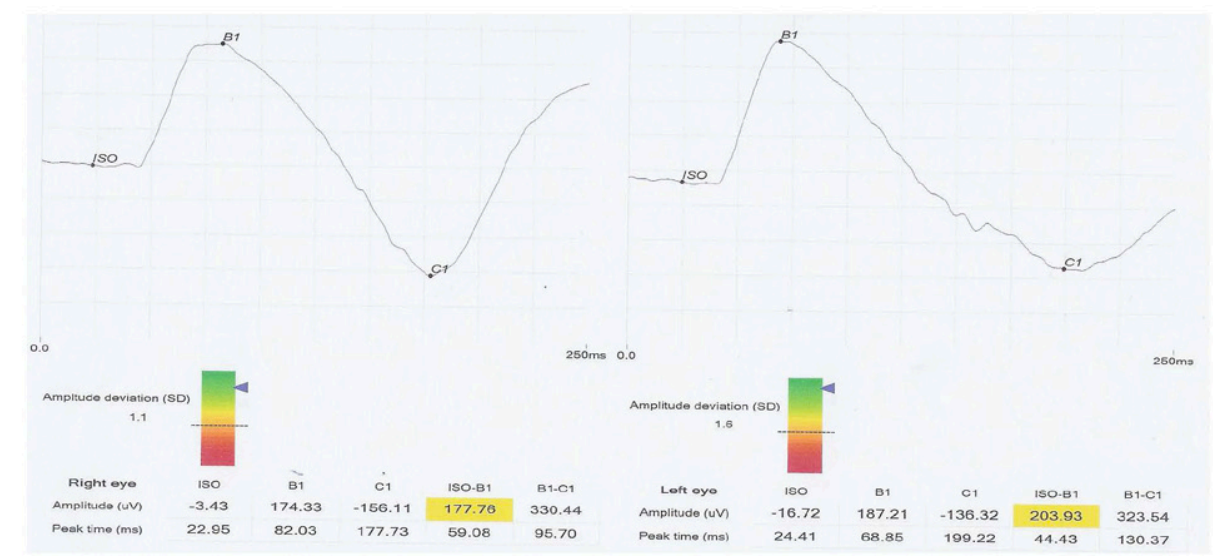

Figure 1: An example of an ERG recording obtained during this study. Included are the ERG plots and the amplitudes and implicit times for the $\mathrm{B}$ and $\mathrm{C}$ peak/troughs for each eye. The position of the $\mathrm{B}_{1}$ peak and $\mathrm{C}_{1}$ trough is indicated.

Figure 2 shows a box and whisker plot of the $\mathrm{B}_{1}$ amplitudes for each subject. The mean and its standard error and the standard deviation for each set of ten measurements are shown and are indicated on the graph by means of a small square, a box, and whiskers (tails) respectively. An indication of the magnitude of the standard error and the standard deviation is given by the size of the box and length of the whiskers.

Tables 2-4 provide the same information as that presented in Table 1 for $\mathrm{B}_{1}$ implicit time, $\mathrm{C}_{1}$ amplitude and $\mathrm{C}_{1}$ implicit time respectively. Figures 3-5 present the $\mathrm{B}_{1}$ implicit times, $\mathrm{C}_{1}$ amplitudes and $\mathrm{C}_{1}$ implicit times (as box and whisker plots) for each subject respectively. Figure 6 shows one example of a graphical representation of the data collected from subject 1 for the un-dilated eye and includes one set of ten measurements.

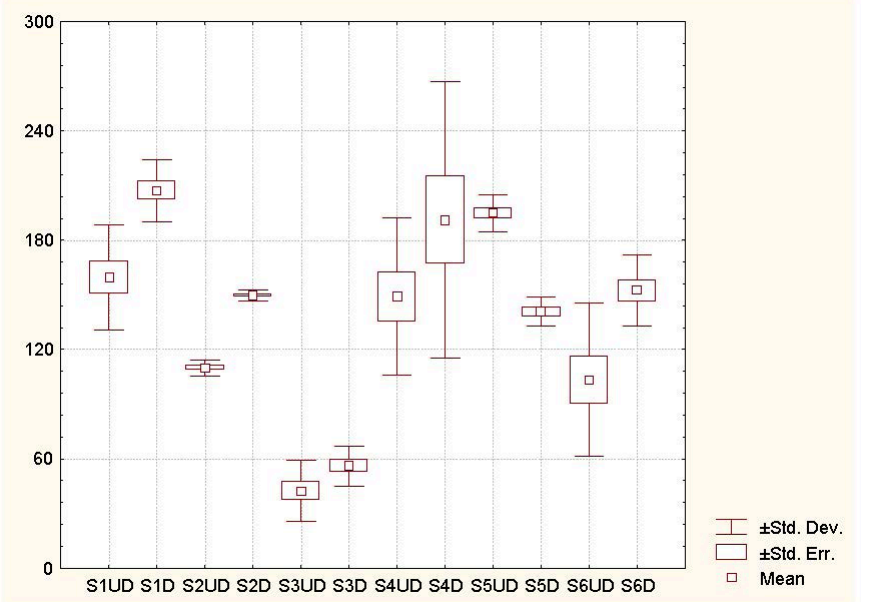

Figure 2: $\mathrm{A}$ box and whisker plot (of $\mathrm{B}_{1}$ amplitudes measured in micro-volts) for each subject is shown. Subjects are labeled S1, S2 etc with UD indicating the un-dilated eye and D indicating the dilated eye. The means, standard errors and standard deviations for all eyes are included.
The variation seen in the ten measurements is representative of other subjects. The descriptive statistics for the data shown in Figure 6 are summarized in Table 1 (subject 1UD). Tables 2-4 provide the consolidated descriptive statistics for the remaining data.

Table 2: The mean $B_{1}$ implicit time, minimum, maximum and standard deviation of ten recordings for each eye of each subject are given. Values are recorded in milliseconds.

\begin{tabular}{l|l|l|l|l} 
Subject & Mean & Minimum & Maximum & Standard deviation \\
\hline 1UD & 159.67 & 97.61 & 189.91 & 28.94 \\
1D & 207.07 & 184.19 & 228.67 & 16.92 \\
2UD & 109.81 & 104.30 & 119.03 & 4.42 \\
2D & 149.61 & 144.77 & 154.46 & 3.06 \\
3UD & 42.47 & -0.51 & 61.88 & 16.57 \\
3D & 56.15 & 38.24 & 77.75 & 11.03 \\
4UD & 149.06 & 58.83 & 225.98 & 43.23 \\
4D & 191.23 & 48.29 & 249.95 & 76.01 \\
5UD & 194.91 & 178.92 & 212.07 & 10.10 \\
5D & 140.92 & 129.88 & 160.29 & 8.15 \\
6UD & 103.53 & 31.25 & 156.02 & 42.01 \\
6D & 152.48 & 114.33 & 177.41 & 19.35
\end{tabular}

Table 3: The mean $\mathrm{C}_{1}$ amplitude, minimum, maximum and standard deviation of ten recordings for each eye of each subject are given. Values are recorded in micro-volts.

\begin{tabular}{l|l|l|l|l} 
Subject & Mean & Minimum & Maximum & Standard deviation \\
\hline 1UD & -73.05 & -180.24 & 28.79 & 59.56 \\
1D & -211.05 & -249.70 & -112.98 & 49.87 \\
2UD & -59.84 & -68.99 & -50.58 & 6.20 \\
2D & -66.10 & -71.31 & -57.70 & 5.56 \\
3UD & -16.66 & -23.00 & -12.81 & 3.92 \\
3D & -10.83 & -25.99 & 13.54 & 14.65 \\
4UD & -93.82 & -168.81 & 39.61 & 43.87 \\
4D & -99.23 & -212.29 & -29.52 & 61.61 \\
5UD & -95.41 & -115.60 & -65.32 & 15.85 \\
5D & -59.86 & -75.98 & 9.90 & 25.86 \\
6UD & -114.13 & -182.26 & 39.65 & 74.13 \\
6D & -10.37 & -107.74 & 78.64 & 52.78
\end{tabular}


Table 4: The mean $C_{1}$ implicit time, minimum, maximum and standard deviation of ten recordings for each eye of each subject are given. Values are recorded in milliseconds.

\begin{tabular}{l|l|l|l|l} 
Subject & Mean & Minimum & Maximum & Standard deviation \\
\hline 1UD & 169.77 & 156.74 & 179.69 & 7.43 \\
1D & 194.09 & 188.96 & 199.22 & 4.36 \\
2UD & 196.53 & 192.87 & 199.22 & 2.58 \\
2D & 196.09 & 190.92 & 199.20 & 3.18 \\
3UD & 196.43 & 190.92 & 199.22 & 2.93 \\
3D & 196.87 & 192.85 & 199.22 & 2.81 \\
4UD & 177.34 & 150.39 & 196.78 & 15.21 \\
4D & 172.51 & 138.18 & 199.22 & 22.66 \\
5UD & 182.42 & 140.14 & 199.22 & 22.25 \\
5D & 178.08 & 121.09 & 187.50 & 20.15 \\
6UD & 175.88 & 126.46 & 197.75 & 23.07 \\
6D & 187.79 & 159.18 & 199.22 & 17.45
\end{tabular}

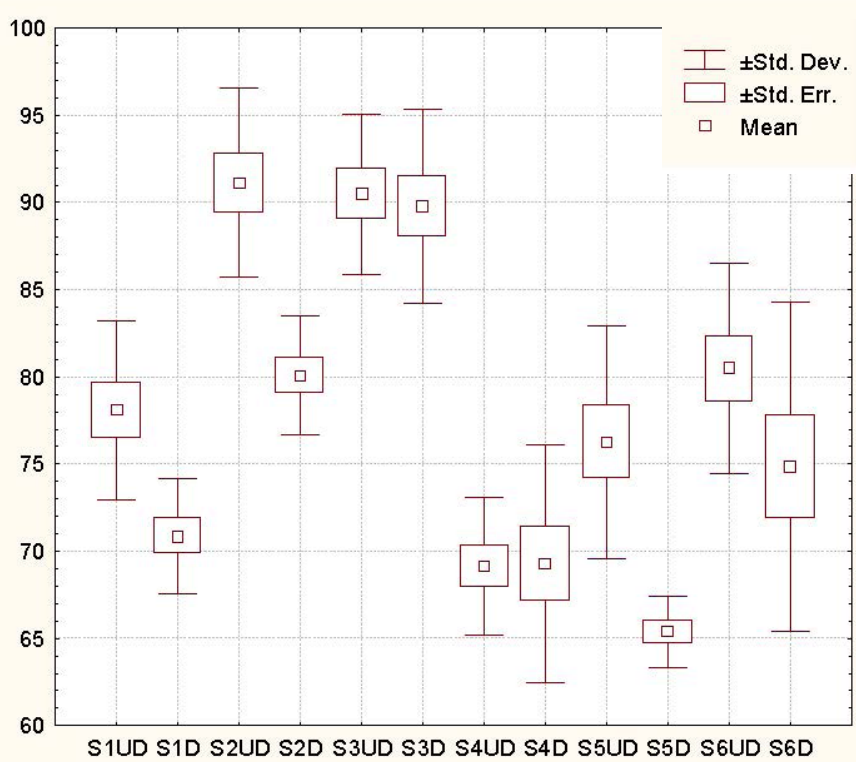

Figure 3: A box and whisker plot (of $\mathrm{B}_{1}$ implicit times measured in milliseconds) for each subject is shown.

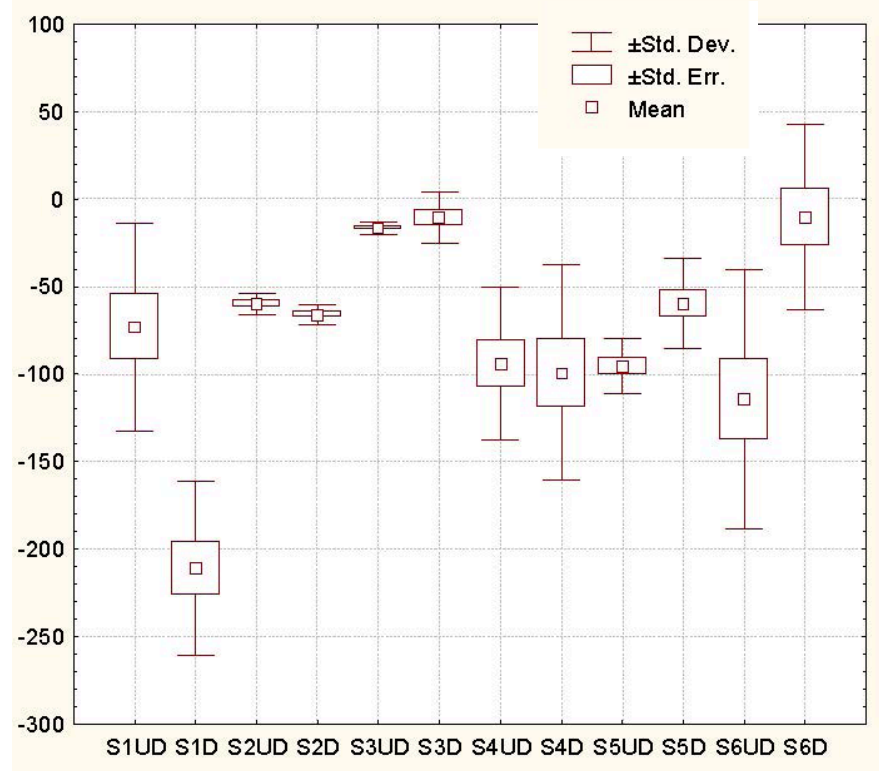

Figure 4: $A$ box and whisker plot (of $\mathrm{C}_{1}$ amplitudes measured in micro-volts) for each subject is shown.
Murray and Lawrence16 emphasize the importance of repeatability of multiple measurements when they state: "Tests with good repeatability are generally better than those with poor repeatability, as they give similar results when repeated on the same patient. However, there is some confusion about how repeatability data should be presented". Bland 17 makes the following observation: "...most clinical measurements cannot be taken at face value without some consideration being given to error". Within subject standard deviation can be used as an indicator of spread of data (variation, and thereby can be an indicator of repeatability), however, the standard deviation needs to be independent of the mean of the set of measurements ${ }^{10}$. Numerous authors make use of the coefficient of variation to further (and in addition to standard deviation) evaluate and/or clarify the level of repeatability of multiple measurments ${ }^{12,15,18-20}$. Bland ${ }^{17}$ defines the coefficient of variation as the standard deviation divided by the mean. The coefficient of variation is a dimensionless number and is more suitable when comparing sets of data which have largely different means. The data collected in this study were subsequently also evaluated using the coefficient of variation.

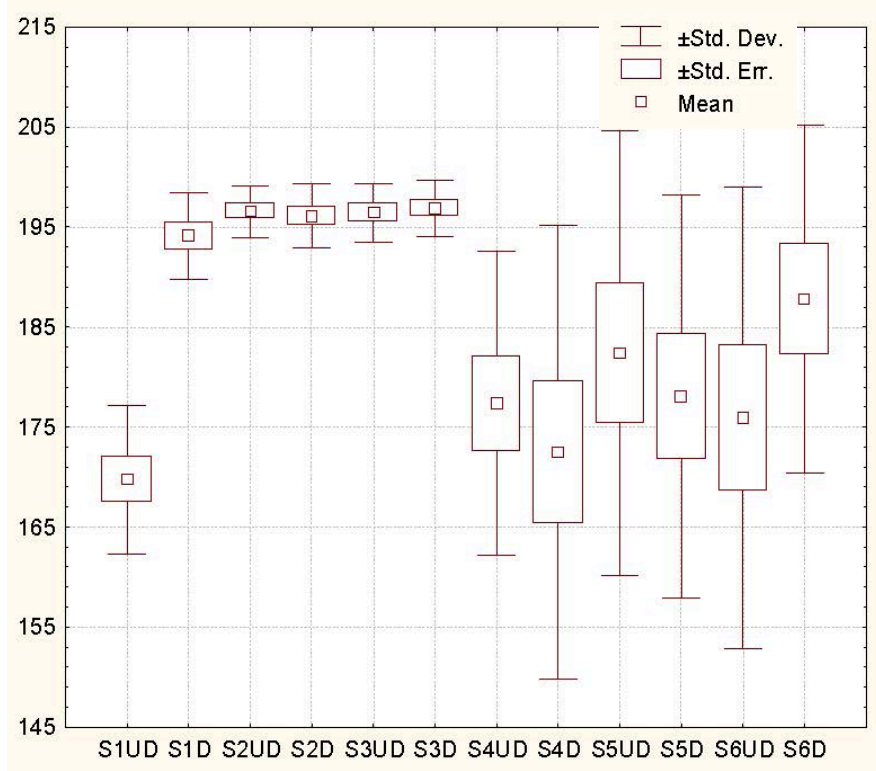

Figure 5: $A$ box and whisker plot (of $\mathrm{C}_{1}$ implicit times measured in milliseconds) for each subject is shown.

Table 5 shows the coefficients of variation for each subject for the amplitudes and implicit times of the B and $\mathrm{C}$ components of the ERGs recorded (some authors indicate the coefficient as a percentage and to 
do so with this data merely multiply each coefficient in table 5 by 100$)$.

Table 5: Coefficients of variation for each subject for amplitude and implicit times.

\begin{tabular}{l|l|l|l|l} 
Subject & $\mathrm{B}_{1} \mathrm{amp}$ & $\mathrm{B}_{1}$ time & $\mathrm{C}_{1} \mathrm{amp}$ & $\mathrm{C}_{1}$ time \\
\hline 1UD & 0.18 & 0.07 & 0.82 & 0.04 \\
1D & 0.08 & 0.05 & 0.24 & 0.02 \\
2UD & 0.04 & 0.06 & 0.10 & 0.01 \\
2D & 0.02 & 0.04 & 0.08 & 0.02 \\
3UD & 0.39 & 0.05 & 0.24 & 0.01 \\
3D & 0.20 & 0.06 & 1.35 & 0.01 \\
4UD & 0.29 & 0.06 & 0.47 & 0.09 \\
4D & 0.40 & 0.10 & 0.62 & 0.13 \\
5UD & 0.05 & 0.09 & 0.17 & 0.12 \\
5D & 0.06 & 0.03 & 0.43 & 0.11 \\
6UD & 0.41 & 0.07 & 0.65 & 0.13 \\
6D & 0.13 & 0.13 & 5.09 & 0.09
\end{tabular}

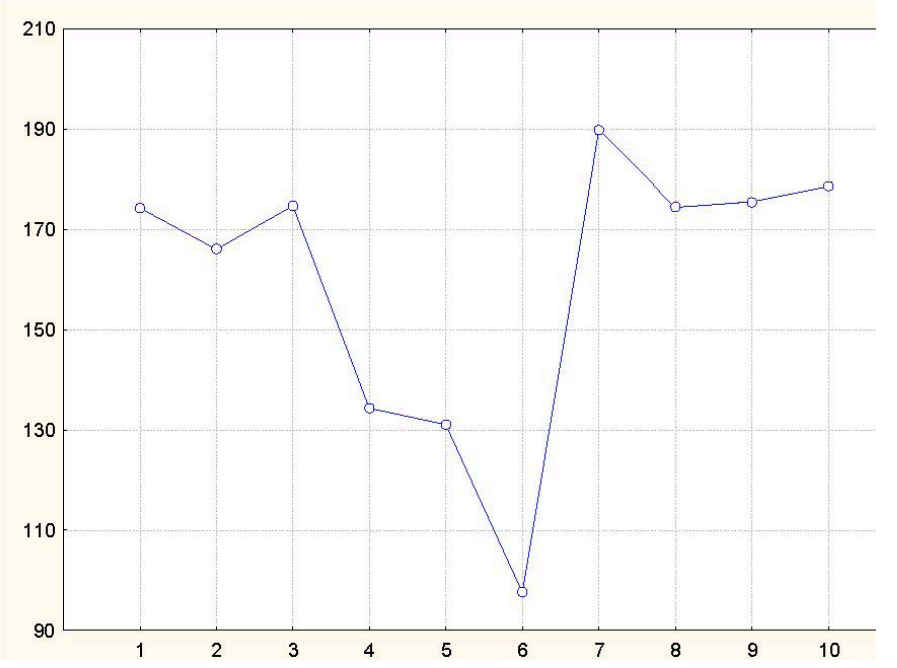

Figure 6: One example of a plot showing the ten data points of $\mathrm{B}_{1}$ amplitude (measured in micro-volts) for subject 1 (un-dilated eye). The data are summarized in Table 1 (subject S1UD). The variation in measurements is representative of other measures. The $y$-axis indicates $\mathrm{B}_{1}$ amplitude while the $x$-axis indicates measurement number. The variability in the measurements is noticeable.

\section{Discussion}

Multiple scotopic flash ERG recordings were obtained from six volunteers in an attempt to quantify the variation (thereby repeatability) that occurs in measurements of this type. Each subject had ten ERG recordings taken (resulting in ten ERG plots per eye) and these were used as data in this study. Data is presented by means of descriptive statistics, box and whisker plots and coefficients of variation. Tables 1-4 and Figures 2-5 present the descriptive statistics and box and whisker plots for the $\mathrm{B}_{1}$ amplitude and implicit time and $\mathrm{C}_{1}$ amplitude and implicit time respectively for each subject. Each box and whisker plot gives a visual indication of the difference in means between the undilated and dilated eye, the difference in standard error as well as the difference in standard deviation. In many instances the differences seem obvious. It needs to be remembered that hypothesis testing was not conducted on this data and so no conclusion can be made regarding whether differences are significant or not. What can be determined from the box and whisker plots is the apparent difference in variation in the data for some subjects (as indicated by the length of the whiskers) as opposed to other subjects as well as the difference within subjects. An example of within subject variation can be seen in Figure 1 where the plots for subject 4 show a large difference in standard deviation between the undilated and dilated eye. The difference between subjects can be appreciated in the plots for subject 2 when compared with the plots for subject 4 . Similar differences can be appreciated in Figures 3-5.

Table 5 presents the coefficients of variation for $\mathrm{B}_{1}$ amplitude and implicit time and $\mathrm{C}_{1}$ amplitude and implicit time for each subject. Taking an arbitrary value of 0.1 as a cut-off point $B_{1}$ and $C_{1}$ amplitudes have more sets of measurements with a coefficient of variation greater than 0.1 indicating that more variation occurred in amplitude measurements than implicit time measurements (also indicating reduced or poorer repeatability). Does this mean that amplitude measurements in scotopic ERGs taken with this instrument need to be considered differently than implicit time? Investigating the effects of different ERG electrodes on variability of measurements Mohidin et $a l^{12}$ showed coefficients of variation to range from 0.15-0.23 while Bayer et al ${ }^{13}$, investigating electrode effects on rodents, found coefficients of variation to range between 0.10 and 0.28 . Coefficients of variation ranging from 0.02 to 7.0 were found by Bartel et al ${ }^{14}$ when investigating the intra-session repeatability of pattern ERG recordings. In another study investigating pattern ERGs Holopigian et a ${ }^{15}$ showed coefficients of variation to extend from 0.22 to 0.41 for different electrode configurations. As indicated in 
the introduction, Holopigian et al ${ }^{15}$ stress the need for caution when using pattern ERG data in the clinical setting as variability of measurements "remained high". Numerous coefficients of variation for subjects in this study are greater than those found in Holopigian et al's study (see Table 5). Other researchers have found similar dispersion of coefficients of variation when investigating inter-session and inter-ocular ERG measurements ${ }^{17,} 18$. In this particular study the data showing the greatest variation in measurements is $\mathrm{C}_{1}$ amplitude followed by $\mathrm{B}_{1}$ amplitude. If one takes an arbitrary value for a coefficient of variation of 0.40 then $\mathrm{C}_{1}$ amplitude shows the greatest variation with the most subjects above 0.40 (see table 5).

Variation in biological measurements is normal and needs to be considered when assessing research and clinical data. This study suggests that ERG amplitude measurements and ERG implicit time measurements, obtained with this CSO electrophysiology system, might need to be approached differently (and with caution) when considering any variation (or repeatability issues) that might occur in repeat measures of scotopic flash ERGs.

\section{References}

1. Carr RE, Siegel IM. Visual electrodiagnostic testing: a practical guide for the clinician. Williams and Wilkins: Baltimore, 1982

2. De Rouck AF. History of the electroretinogram. In: Principles and practice of clinical electrophysiology of vision 2nd ed. Heckenlively JR, Arden GB eds. The MIT press: Massachusetts, 2006 3-10.

3. Millodot M. Dictionary of optometry and vision science. 4thed. Oxford: Butterworth-Heinemann, 1997.

4. Cline D, Hofstetter HW, Griffin JR. Dictionary of visual science. 4th ed. Oxford: Butterworth-Heinemann, 1997.

5. Parisi V, Uccioli L, Monticone G, Parisi L, Manni G, Ippoliti D, Manzinger G, Bucci MG. Electrophysiological assessment of visual function in IDDM patients. Electroencephalo Clin Neurophysiol 1997104 171-179.

6. Holder GE. Pattern electroretinography (PERG) and an integrated approach to visual pathway diagnosis. Prog Ret Eye Res 200120 531-561.

7. Hopkins WG. A new view of statistics. http://www.sportsci. org/resource/stats/precision .html. Accessed June 2011.

8. Taylor BN, Kuyatt CE. Guidelines for evaluating and expressing the uncertainty of NIST measurement results. NIST technical note 1297, United States Department of Commerce, Technology Administration, 1994.
9. Bland JM, Altman DG. Statistics notes: measurement error. BMJ 1996313 744-745.

10. Bland JM, Altman DG. Statistics notes: measurement error proportional to the mean. BMJ 1996313106.

11. Hess RF, Baker CL, Verhoeve JN, Keesey UT, France TD. The pattern evoked electroretinogram: its variability in normals and its relationship to amblyopia. Inv Ophthalmol Vis Sci 198526 1610-1623.

12. Mohidin M, Yap MKH, Jacobs RJ. The repeatability and variability of the multifocal electroretinogram for four different electrodes. Ophthal Physiol Opt 199717 530-535.

13. Bayer AU, Mitttag T, Cook P, Brodie SE, Podos SM, Maag K-P. Comparisons of the amplitude size and the reproducibility of three different electrodes to record the corneal flash electroretinogram in rodents. Doc Ophthalmol 2000 98 233-246.

14. Bartel P, Becker P, Robinson E. The intrasession repeatability of pattern electroretinograms and the effects of digital filtering. Doc Ophthalmol 199176 351-358.

15. Holopigian K, Snow J, Sieple W, Siegel I. Variability of the pattern electroretinogram. Doc Ophthalmol 198870103 115.

16. Murray A, Lawrence GP. How should the repeatability of clinical measurements be analyzed? An assessment of analysis techniques with data from cardiovascular autonomic tests. Quarterly Jnl Med 199386 831-836.

17. Bland M. An introduction to medical statistics. Oxford: Oxford Medical Publications, 2000, 270-271.

18. Fortune B, Bui BV, Cull G, Wang L, Cioffi GA. Inter-ocular and inter-session reliability of the electroretinogram photopic negative response ( $\mathrm{PhNR}$ ) in non-human primates. Exp Eye Res 200478 83-93.

19. Bui BV, Fortune B, Cull G, Wang L, Cioffi GA. Baseline characteristics of the transient pattern electroretinogram in non-human primates: inter-ocular and inter-session variability. Exp Eye Res 200377 555-566.

20. Sidhu PS, Desai SR. A simple and reproducible method of assessing intimal-medial thickness of the common carotid 